\title{
Reach out and touch: using NFC and $2 D$ barcodes for service discovery and interaction with mobile devices
}

\author{
Eamonn O’Neill ${ }^{1}$, Peter Thompson ${ }^{2}$, Stavros Garzonis ${ }^{1}$ and Andrew Warr ${ }^{1}$ \\ ${ }^{1}$ Department of Computer Science, University of Bath, UK, BA2 7AY \\ \{eamonn, s.garzonis, cspaw\}@cs.bath.ac.uk \\ http: //Www. cityware.org.uk \\ ${ }^{2}$ Vodafone Group R\&D, Newbury, UK, RG14 2FN \\ peter.thompson@vodafone.com
}

\begin{abstract}
We investigate the use of 2 tagging technologies: Near Field Communication (NFC) and 2-dimensional barcodes. Our investigation combined a field trial and interview based study with an experimental evaluation. The field trial focused on users' experience of the usability of NFC for a range of trial services, users' perceptions of NFC use in their daily life context, and users' suggestions for potential applications of NFC. The tags were embedded in a variety of postcards, table-top signs and posters. The experimental evaluation compared the ease of use of NFC and 2D barcodes, operationalised in terms of time taken to read a specified sequence of tags on posters. We found that for untrained users the 2D barcodes were quicker to use than the NFC tags but that training significantly improved users' performance with the NFC tags while having no effect on their performance with the barcodes.
\end{abstract}

\section{Introduction}

The use of ambient tags read by mobile devices presents new possibilities for service discovery and user interaction. Service discovery is an increasingly important issue as we move towards realising pervasive systems. How does the user identify and access a particular service from the plethora of services that may potentially be available around him at any moment? Having discovered a service, a second important issue is how the user interacts with it. Tags in the environment may be used to provide fast, zero-configuration service discovery and support interaction between mobile and fixed devices in pervasive systems [8]. Tags may be used to store small amounts of data on the tag itself. However, more typically, the tag may store a link to more sophisticated information and services. The link may connect the tag-reading device to a URL, email address or phone number, or may provide a unique identifier as a reference to information or services. Two very different tagging technologies, 2D barcodes and NFC, are each beginning to see widespread interest but as yet there are few reported studies of either in use [10].

2D barcodes can be easily produced and printed. They can store more information than the currently ubiquitous 1D barcodes but, unlike 1D barcodes, have not yet been standardised. Recent software applications enable any device with a camera, such as a mobile phone, to read 2D barcodes with no additional hardware required. A 2D 
barcode may be printed on a surface or on an adhesive tag which may in turn be attached to a surface. Alternatively, a 2D barcode may be displayed on a device's screen while another device is used to read the tag. A 2D barcode provides one-way communication. The barcode image encodes data. The mobile phone's camera scans the image and the reader application decodes the data from the image. Typical uses include stock control but also e-ticketing (e.g. airline boarding passes) and triggering mobile data services from a location or object [e.g. 4]. [12] used a form of 2D barcode on posters and active displays to establish wireless connections between mobile phones and site-specific services. This work integrated previous work [11] that used the barcodes for device discovery to bypass the Bluetooth scanning protocol, significantly reducing discovery time.

NFC is a short range wireless technology, similar to other RFID technologies but with additional features (see www.nfc-forum.org). NFC devices operate in reader/writer mode, peer-to-peer mode, or card emulation mode, based on the ISO/IEC 18092 NFC IP-1 and ISO/IEC 14443 standards. In reader/writer mode, an NFC device can read or write NFC tags similar to the uses of standard RFID tags. In peer-to-peer mode, two NFC devices can directly exchange data, simply by touching the devices together to initiate a wireless link. NFC can be used to initiate a channel between devices and then hand over to complementary wireless technologies such as Bluetooth and WiFi. In card emulation mode, an NFC device appears to an NFC reader as an NFC tag with secure data storage capacity, allowing the device to be used for applications such as contactless payment and eticketing.

The market for RFID systems is currently driven by traditional applications such as access control, car engine immobilization, automated toll collection, and stock control and transportation. NFC has the potential to drive new applications and markets [13]. NFC devices are currently in use in the form of simple payment cards like the Oyster card used by Transport for London in the UK. In the US, NFC use has so far been confined to the development of NFC-enabled credit cards. The major mobile phone manufacturers are committing to NFC. Nokia got to market first with an NFC shell for its 3220 phone, which we used in the studies reported here. Although the use of NFC-equipped mobile phones is currently still in its infancy, a study by ABI Research predicts that $50 \%$ of all mobile phones will support NFC by 2009 , with sales of NFC phones exceeding 500 million by $2010^{1}$. The study also predicts that by 2007, NFC deployments will be common in many kinds of consumer electronics, from computers to cameras, printers, set-top boxes etc.

NFC tags and 2D barcodes require slightly different use. NFC requires the user to bring her device very close to the tag. 2D barcodes require the user to point her camera at the tag (and with some readers, take a photo). NFC offers secure data storage features that 2D barcodes cannot offer. In general, however, NFC and 2D barcodes support many of the same services. Over the next few years, it seems likely that NFC and 2D barcodes will offer alternative means of accessing some of these services, such as eticketing (where the data may be stored securely in an NFC device or dis-

\footnotetext{
${ }^{1}$ ABI Research (2005) Near Field Communications (NFC): simplifying and expanding contactless commerce, connectivity and content. Report RR-NFC. Available at http://www.abiresearch.com/products/market_research/Near-Field_Communications_(NFC). Last accessed 13 October 2006.
} 
played as a barcode on the user device's display, in either case being read by an external reader) and service discovery (where NFC tags or 2D barcodes are printed on or attached to objects in the environment).

In the work reported here, we investigated the use of tagging to support service discovery and interaction with mobile phones using NFC and 2D barcodes. In the first study, we conducted a field trial using NFC in which most of the services offered, other than writing personalized tags directly from the phone, could equally have been supported by 2D barcodes. In the second study, we directly compared the use of NFC and 2D barcodes in a lab-based experimental evaluation. Our field trial provided empirical data on the popularity of various tag based services, user feedback on the use of NFC phones and tags, and suggestions for NFC applications. The field trial did not attempt to distinguish between NFC and 2D barcodes as appropriate tagging technologies. This was investigated in our second study, in which we conducted an experimental comparison of NFC and 2D barcode use.

\section{NFC field trial}

The main objective of the field trial in our first study was to gather user feedback on the use of NFC to discover and interact with mobile services. In particular, we were interested in (i) users' experience of the usability of NFC for a range of trial services, (ii) users' perceptions of NFC use in their daily life context, and (iii) users' suggestions for potential applications of NFC.

\subsection{Participants}

A total of 60 Vodafone employees from various departments volunteered for the trial. Ages ranged from 22 to 51 years of age, with a median of 32 and a mean of 31 . Several volunteers were unavailable at short notice so only 55 participants completed the opening questionnaire, 36 male and 19 female. Throughout their regular workdays the participants are exposed in varying degrees to the development, use or marketing of mobile phone technologies. They are relatively knowledgeable technically and are experienced in using new features on mobile phones.

\subsection{Equipment}

A total of 1226 NFC tags were used in the field trial. Prior to the study, table-top signs and A3-size wall posters were distributed in 40 locations across six buildings, including business centres, meeting areas and kitchens. NFC tags were embedded in the posters and signs. The services accessed by reading a tag ranged across the services offered by mobile phone operators: voice, data and SMS. There were 90 tabletop signs, each containing tags with links via URLs to Vodafone Live! sport, music and games sites. 16 of the posters had a variety of tags. For example, the "Local traffic news" poster (see Figure 1) had a tag that triggered an SMS request for infor- 
mation on local traffic conditions. The response was sent by SMS to the user's phone. A second tag on this poster linked to the Vodafone Live! traffic news site.

A "Music" poster contained a tag that linked to the Vodafone Live! music site, and a second tag that entered the user in a prize draw competition with a CD as the prize. A "Sport" poster contained a tag that linked to the Vodafone Live! sports site, and a second tag that entered the user in a prize draw competition with tickets to a football game as the prize. A further 20 posters contained tags that formed a "treasure hunt". Participants who managed to read all the tags embedded in the posters with a treasure hunt icon were entered in a prize draw for a miniature Ferrari.

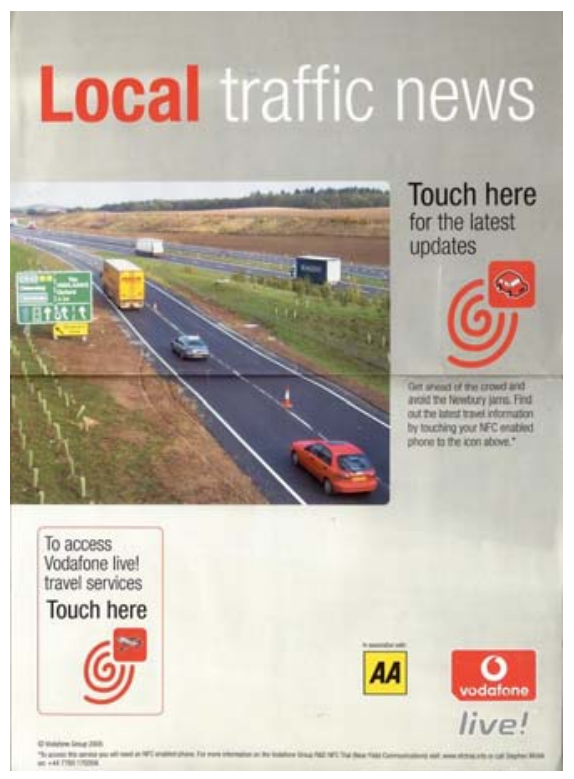

Fig. 1. A poster with embedded NFC tags.

A trial pack was also given to each participant at the start of the trial. The pack included a Nokia 3220 phone with NFC shell, a set of postcards each with an embedded tag, and an introductory letter with 2 embedded tags. One linked to a WAP-based site offering information about the trial and NFC. The other triggered a voice call from the user's phone to a "trial helpline" supported by the trial administrators. One postcard tag downloaded free wallpaper for the user's phone. The wallpaper offered changed daily. Another postcard tag downloaded a free ringtone for the user's phone. Similarly, the ringtone offered changed daily. Three "Keep in touch" postcards had "blank" tags. Participants were encouraged to write their own content (e.g. text messages, URLs or phone numbers) to these tags and leave the postcards around for others to find and read. Finally, a "directory assistance" postcard triggered a voice call from the user's phone to the main Vodafone directory assistance service. 


\subsection{Procedure}

The trial lasted 9 days, beginning on a Thursday and running through to the following Friday. On the first day, the participants were given an introductory briefing and a trial pack. 55 of the participants completed a pre-trial questionnaire. This covered the participant's usage of mobile phones, including voice, text and data services and phonecam use; their usage of the Internet, including email and web use; and their wishes for new mobile applications and features.

The services were activated from the first day of the trial (Thursday) with the exception of the "treasure hunt" which began on the fifth day (Monday). The trial administrators and 17 "wizards" (staff who were very familiar with the technologies) were available to provide help and advice to the participants throughout the study.

The data services were delivered via an NFC server implemented for the trial. However, this server was not involved in interactions with the travel information SMS tags, since the SMS transmissions bypassed the NFC server, or the interactions that accessed a voice service (i.e. reading the "trial helpline" and "directory assistance” tags), in which a voice call was placed directly to the service provider.

At the end of the trial, 47 of the participants completed a post-trial questionnaire. This covered their experiences during the field trial, and their comments and suggestions for future applications and uses of NFC based services. The questionnaire was followed up with an interview.

\subsection{NFC field trial results}

\subsubsection{Tag usage}

The NFC server log provided a quantitative measure of a subset of the participants' interactions with the NFC system, i.e. excluding voice calls and SMS messages. A total of 2005 tag interactions by participants were logged by the server during the first 8 days of the trial. (Tag events were not logged for the final day of the trial.) Table 1 shows the number of different tags accessed by a participant, i.e. not counting repeated accesses of a particular tag by the same participant.

Table 1. Distribution of data service interactions with different NFC tags.

\begin{tabular}{cc}
\hline Distribution of tags & Number of participants \\
\hline 0 & 04 \\
$1-10$ & 35 \\
$11-20$ & 16 \\
$21-30$ & 02 \\
$31-40$ & 03 \\
\hline
\end{tabular}


Participants read a high number of tags in the first 2 days after receiving their NFC phones and trial packs (Thursday and Friday). 64 tag events were recorded during the weekend despite a network problem that became apparent on Friday. The trial administrators sent an email early on the Monday morning, informing the participants that the problem had been fixed. Over half the total tag interactions were then logged during the Monday and Tuesday. Tag events decreased over the next two days (Figure 2).

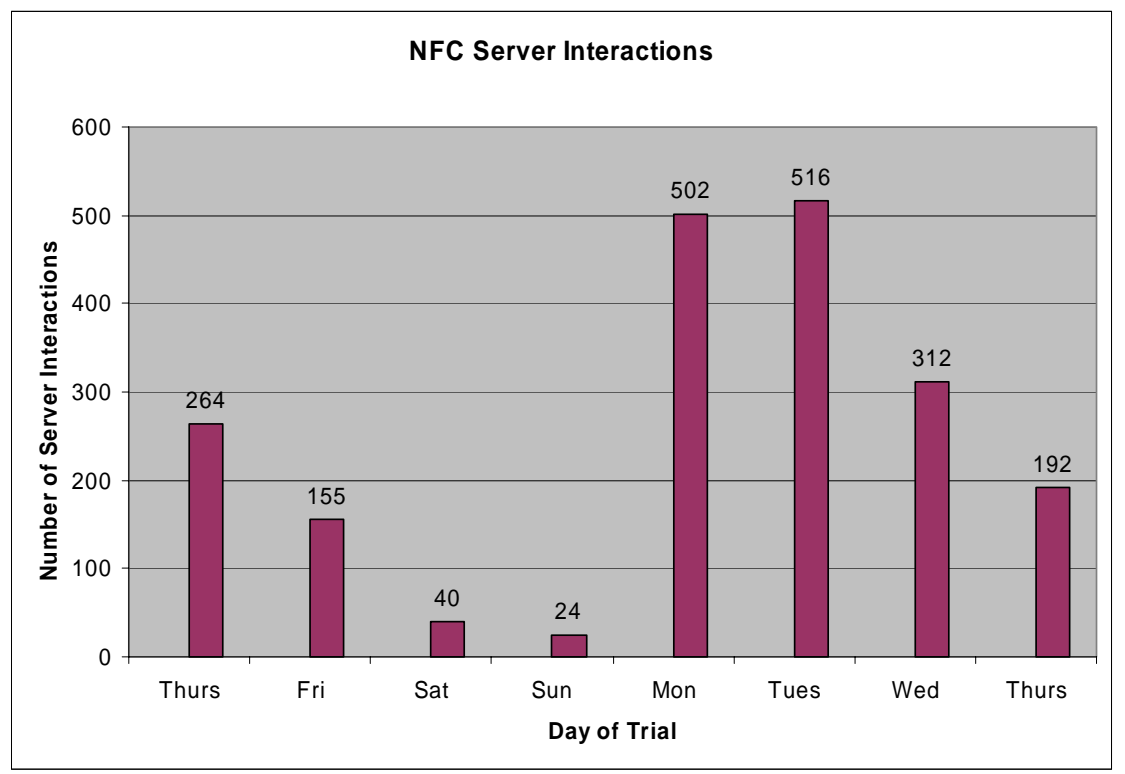

Fig. 2. NFC server events per day.

Table 2 compares the use of the various tags for the days when the treasure hunt was active. Content downloads (free daily wallpapers and ringtones) were the most popular tags, followed by tags that accessed the Vodafone Live! information sites.

Table 2. Tag usage for days when the treasure hunt was active.

\begin{tabular}{llll}
\hline & Min/day (Mon-Thu) & Max/day (Mon-Thu) & Total (Mon-Thu) \\
\hline Content downloads & 58 & 168 & 464 \\
Vodafone Live! information & 34 & 166 & 404 \\
Competitions & 22 & 68 & 174 \\
Treasure hunt & 64 & 194 & 398 \\
Trial Information WAP site & 2 & 36 & 82 \\
\hline
\end{tabular}




\subsubsection{User experience with NFC trial services}

The participants' extensive experience with mobile phones helped make them comfortable using the NFC phones. Occasional problems with the software were overcome by restarting the phone, participants developed workarounds for unimplemented menu options, and NFC tag reading was approached with more patience than might be the case with less experienced users.

Although $96 \%$ of participants regarded the NFC symbols indicating the locations of the tags on the posters, signs and postcards as easy to find and identify, and $90 \%$ claimed that the location of the NFC reader in the phone was obvious, only $40 \%$ felt that the use of the reader was clear from the start. Most reported that they had to experiment with reading tags, as it was not clear if one had to hover, slide, wave or press the reader on to the tag.

After an initial period of experimenting with the NFC interaction process, participants developed their own ways of reading a tag. Different users reported different techniques, including touching the tag with the top of the phone, sliding the phone across the tag, and holding the phone $3 \mathrm{~cm}$ from the tag. Participants became convinced that their device responded best to a particular angle, motion or distance from the tag. After the initial experimentation, most participants found the process of reading tags straightforward and intuitive, although $65 \%$ of participants reported occasional problems reading tags, $26 \%$ reported frequent problems and only $9 \%$ reported no problems.

$25 \%$ of participants regarded the speed of accessing services as good, $37 \%$ as adequate and $37 \%$ as too slow. These should be interpreted as the perceptions of very experienced mobile phone users, accustomed to delays in downloading data services on pre-3G networks.

Some participants found that the screensaver and keyboard lock features on the phone interfered with the use of NFC. One could read a tag with the screensaver or the keyboard lock enabled, but had to unlock the keyboard before the corresponding service could be accessed or viewed on the screen. Given that reading an NFC tag is intended to require an explicit user action, participants felt that reading a tag should automatically turn off the screensaver and keyboard lock.

Half of the participants wrote to their "Keep in touch" tags, mainly storing contact details or URLs on the tag. The remaining participants did not write to the tags. The follow up interviews revealed that some of these participants attempted to but were unable to complete the tag writing successfully.

Privacy and security concerns arose during the trial and were discussed during the post-trial interviews. While some participants did not perceive any security problems with the technology, others were more concerned. Many of the latter were wary of the "always on" status of the NFC reader in the phone. At times the NFC phone would unintentionally interact with nearby tags, e.g. when a tagged postcard was next to the phone in the participant's bag.

Participants were also concerned that tags could advertise one service, for example through a poster, while actually connecting to another, perhaps at a premium rate. This could be perpetrated deliberately by the producer of the poster. Even more insidiously, a poster offering a legitimate service could be subverted by having its tag 
rewritten. Participants were also concerned that rewriting tags could be used to create a nuisance for third parties via the reader's phone. By writing a message to a tag that included the third party's number, one could encourage people reading the tag to phone the third party, with potentially unwelcome results. Worse still, one could write the tag to trigger a call directly from the reader's phone to the third party. Perhaps more subtly, one participant suggested, "I could write someone else's name to a tag and link it to an inappropriate website or include an impolite message and leave it for his manager [to read the tag]”.

\subsubsection{NFC in the users' daily life context}

The potential of a tag-based system to identify precisely an individual's location and activities is sometimes viewed as a threat to people's privacy [1; 6]. Our participants regarded this issue as relatively unimportant. Participants felt that the devices they currently use - swipe cards, computer log-ins and so on - already record their locations and activities sufficiently closely that reading some NFC tags would not introduce a significant extra opportunity for monitoring their behaviour. To paraphrase Scott McNealy, these users have lost their privacy and got over it.

Participants were more concerned with how the use of NFC readers in public spaces made them appear to other people around them. Many participants noted that they felt awkward at first using NFC due to the very explicit public act of reaching out and touching a tag embedded in a poster or sign. However, despite describing it as "odd" to use at first, many participants lost their reservations about using NFC over the course of the trial.

While the process became more familiar to participants, their interactions with the tags remained objects of curiosity to many of their colleagues. (This became clear when the trial administrators began to receive requests to be included in the trial from people who had noticed the participants reading tags.) The very requirement to make NFC tags both noticeable and accessible makes the reader-tag interactions public.

When asked if they would use NFC in public places, 13 participants responded that the nature of the place was a determining factor. In high traffic public places where people often seek information (e.g. an airport or train station concourse), they would use NFC. In low traffic areas they would be less likely to use NFC. Even in high traffic places, participants suggested that the interaction time with tags - and potential for consequent interaction with other people in the vicinity - should be minimised. The location of some tags during the trial was regarded as intrusive. In cases of repeated tag reading errors, the trial participant would be blocking the access to a photocopier, for example, thereby interfering with other people's work. And of course if many people wanted to interact with a particular tag, long interaction times would cause another form of blocking, particularly in high traffic places.

In addition to the problem of blocking others' movements and activities, participants voiced another concern with the use of NFC in public places. When an NFC user buys a ticket for a concert, for example, by touching a public poster, a bystander observing the interaction could in principle acquire information about the user's shopping and leisure preferences. However, it seems unlikely that a would-be pro- 
filer following NFC users around would be able to gather sufficient reliable simply from observation.

Many participants were concerned that reaching out to read a tag in public was openly advertising one's phone, potentially catching the attention of thieves. There was a perception amongst participants that busy places are potentially high crime areas, and using NFC in such places was regarded as potentially dangerous. In response to this perceived threat, participants suggested that tags might be placed where users could read them close to their body, without reaching out.

\subsubsection{Suggested NFC applications and services}

In addition to providing feedback on the trial services, participants suggested potential applications for NFC. In addition to existing NFC services, such as mobile electronic payment, their suggestions included applications in:

- access control;

- $\quad$ using mobile services; and

- data storage, retrieval and transfer.

Participants commented that they currently gain access to their offices, meeting rooms etc by the use of passive RFID swipe cards. NFC was seen as a valuable alternative, the notion of carrying one device fewer appealing to participants. Embedding access control into the mobile phone was regarded as adding a level of security since our participants believed that people neither leave their phone at home very often nor lend their phone to other people. The little empirical evidence that has been published tends to support this assumption [7]. The risk of losing the phone, and the consequent ability for others to access one's office, was initially viewed by some participants as dangerous. However, they noted that losing a swipe card has the same inherent problem. With NFC, there is the potential to add security features such as deactivation of the sim, access rights, credit card etc with a single action.

A typical NFC service proposed by the participants was ordering a taxi by reading a dedicated tag in a restaurant or shop. Similarly, using tags at bus stops to invoke an SMS message giving the arrival time of the next bus was proposed. At Vodafone HQ, a similar service already exists for the shuttle bus to nearby Newbury, but it is invoked by an SMS sent by the user. Many participants commented that they do not use this service because they do not know the number to which to send their SMS. A shuttle-tag in the HQ lobby could be used to inform users of the time of the next shuttle's departure, simply by touching the tag.

The most commonly suggested application of NFC was contact information management, with the NFC phone being used to store and transfer business card details. In one proposal, an NFC tag could be embedded in a business card, with the recipient of the card reading the tag to transfer the data to her phone's contacts list. Another proposal was the direct interaction of two NFC phones. By touching the phones to each other, the users' contact details could be transferred between the phones.

Another proposal for managing and using data was to "bookmark" physical objects with tags. Rather than trying to remember the details of a friend's CD, one could 
simply read its tag and store it in a "Favourites" folder on the phone. This information could then be used to link to, for example, online retailers or review sites.

Overall, our field trial gave us some interesting empirical data on the popularity of various tag based services amongst our participants, provided user feedback on the use of NFC phones and tags, and provided suggestions for NFC applications. Almost all of these services and applications could be supported by either NFC tags or 2D barcodes, or indeed a combination of both. (The exceptions include electronic payment where the NFC technology is augmented with secure data storage, and the facility to write directly to tags from the phone.) The field trial did not distinguish between NFC and 2D barcodes as appropriate tagging technologies. Our second study filled this gap with an experimental comparison of NFC and 2D barcode use, in which we employed 2D barcodes with the same physical dimensions as the NFC tags.

\section{Experimental comparison of NFC and 2D barcodes}

Our experimental comparison of NFC and 2D barcodes reflected the use of posters with embedded tags in our field trial. However, in order to control confounding variables such as poster content, the posters in the experiment were as simple as possible, containing just the number of the poster and the embedded tags (see Figure 4). We were interested in the ease of use of the tags themselves, comparing NFC against 2D barcodes, rather than people's experience with the poster content and linked services that were the focus of our field trial.

Therefore, we provided the same, very simple "service" from every tag. Successfully reading a tag provided the user with an instruction on the phone to move on to the next tag. The main difficulty with using the tags in our field study, and in the pilot for our experimental study, was repeated unsuccessful attempts to read a tag. Hence, time taken to read the complete set of tags in the correct sequence was a useful and reliable quantitative comparison of the 2 forms of tagging.

In both the field study and the experimental pilot, we experienced the problem of users not knowing the precise location of the NFC reader in the phone. With multiple adjacent NFC tags, this resulted in users repeatedly scanning the wrong tag because they aimed the wrong part of the phone at the target tag, causing the reader to read an adjacent tag. With multiple adjacent $2 \mathrm{D}$ barcodes, the reader had difficulty in interpreting a single tag when parts of more than one barcode appeared in the camera's viewfinder, so distance to the tag and image composition became important.

To investigate these effects in our main experiment, we arranged multiple tags in different patterns on the posters and varied which of the group of tags the user was required to read.

In our experimental pilot, we found that the fastest $2 \mathrm{D}$ barcode reading was achieved with the phone approximately $100 \mathrm{~mm}$ from the tag, in contrast to the optimal distance of about $20 \mathrm{~mm}$ found with the NFC tags. The angle of the phone also had a marked effect on 2D barcode reading. The phone had to be reasonably parallel to the plane of the barcode for successful reading (although this problem could be mitigated in software by the reader). To investigate this effect in our main experi- 
ment, we arranged the posters at different heights to change the angle of incidence to the tags.

Overall, our pilot study suggested that using 2D barcodes was at first easier than using NFC, with fewer of the initial difficulties and need for experimentation with the NFC reader also found in our field study. However, the difference in usability between the 2 technologies lessened quite quickly as users became more experienced with the NFC reader and settled on a particular way to handle and aim the reader. This reflected our findings from the field study.

\subsection{Experimental design}

Our experiment had a mixed factorial design. There were two factors, each of which had two levels. The independent variables were: (i) whether 2D barcodes or NFC tags were used; and (ii) whether the participants were given training or not. The dependent variable was the time it took a participant to scan all the indicated tags in the correct sequence. It was predicted that for untrained users the 2D barcodes would be quicker to use than the NFC tags (H1). It was also predicted that training would significantly improve users' performance with the NFC tags (H2).

\subsection{Participants}

Twenty-eight participants took part in the experiment, 20 male and 8 female. The participants varied in age from 19 to 30 years of age, with a mean age of 21 years (SD 2.8). The participants were all university students. The participants were recruited via mailing lists and word of mouth, and volunteered to participate in the experiment.

\subsection{Equipment}

The NFC tags and the 2D barcodes were located on the bottom of an A3 poster. 12 posters were attached to a wall in a usability lab (Figure 3). Each poster had a large number printed in the centre ranging from 1 to 12 . Each poster had either 1 or $32 \mathrm{D}$ barcodes in the bottom left corner and the same number and layout of NFC tags in the bottom right corner.

The posters were arranged in numerical order in four columns and three rows. The numbering of the posters went from 1 in the top-left corner through to 12 in the bottom-right corner, in an across-then-down order. Posters in a row were placed so that the target tags were horizontally aligned with each other, to present consistent target heights of 210, 150 and $90 \mathrm{~cm}$. 150cm represented a comfortable reaching out height for a standing person. Based on our pilot study findings, $210 \mathrm{~cm}$ and $90 \mathrm{~cm}$ were used to test usability for standing users when presenting the phone close to and parallel to the plane of the tag was less easy than at shoulder height. $90 \mathrm{~cm}$ also represented a 
standard height for wheelchair-accessible facilities such as ATMs. $210 \mathrm{~cm}$ also reflected experiences of regulatory requirements on the placement of NFC tags in a wide area installation [4].

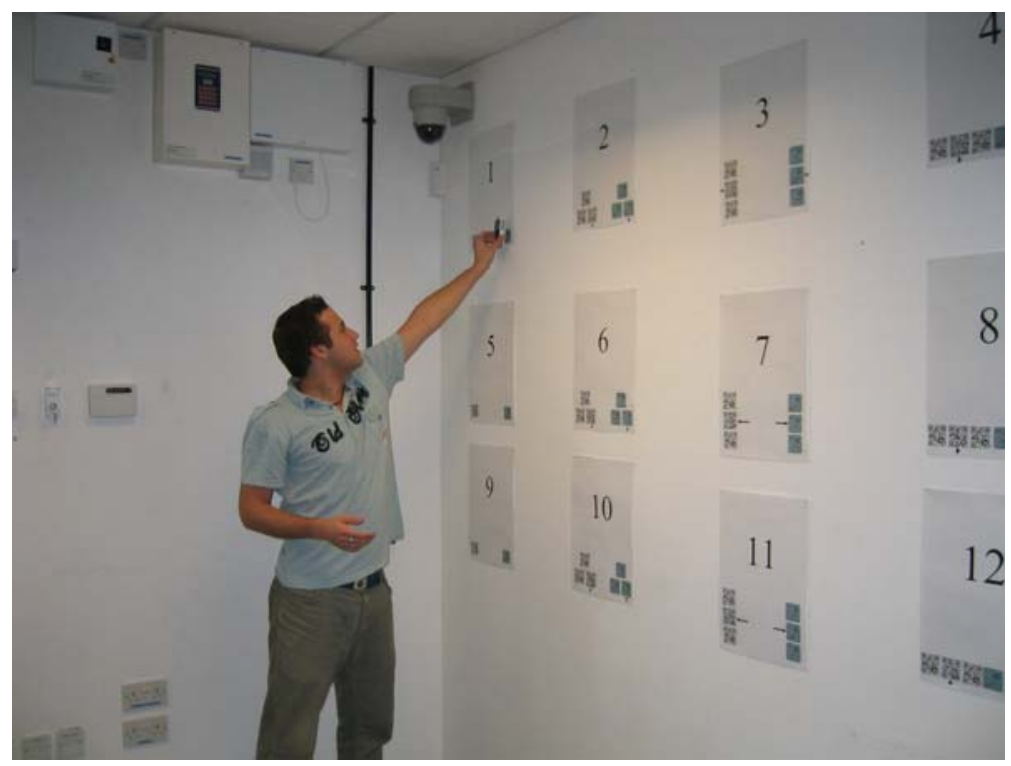

Fig. 3. Reading an NFC tag on a poster.

Across the four columns a variety of tag arrangements was used. As noted above, pilot testing revealed that different arrangements of tags could cause erroneous interactions. The arrangements of tags in our main experiment obliged the user to select the appropriate tag and not merely wave the tag reader around until a tag was read. The target tag in each group of 3 tags was indicated by an adjacent arrow. The groupings of the tags are illustrated in Figure 4, with the target tag highlighted in each group.

a)

c)

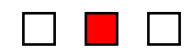

b)

d)

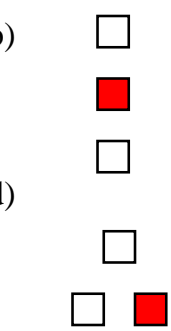

Fig. 4. The arrangements of NFC tags and 2D barcodes on the posters.

The 2D barcodes were read using a Nokia 6600 mobile phone running the Glass 2D barcode reader application (http://www.activeprint.org/). The NFC tags were read using a Nokia 3220 with an integrated NFC reader. Ideally we would have liked 
to use the same phone, however hardware incompatibilities prevented this. The differences between the phones were negligible for our purposes.

When running the Glass application, the view from the camera is shown on the phone's display. When the camera is pointed at a 2D barcode, the application automatically decodes the information held in the barcode and displays it on the screen of the phone as a scrolling message. In our experiment, the message told the user which poster to go to next.

To read the NFC tags, we used Nokia's Service Discovery application. When this is running, it displays the message "Please touch tag" on the phone's display. When the reader is placed close to an NFC tag, the application reads the information held on the tag. This is then displayed as a message on the phone's screen. As in the 2D barcode condition, the message told the user which poster to go to next. Because the Service Discovery application is designed to connect to a URL, the user is also given options to "Connect" or to "Cancel" each time a tag is read. For the experiment, we limited the user option to just "Cancel”. Pressing the "Cancel” button returned the phone to "Please touch tag", ready to read the next tag.

Using either of the technologies, when a tag was read that was not the current target tag, a message appeared on the phone's display informing the user that an incorrect tag had been read.

During the experiment, 3 video cameras recorded the trials. 2 cameras built in to the usability lab captured a wide and a high view respectively of the user interacting with the tags, while a tripod mounted camera captured the view from behind the user facing the posters on the wall. An observer also took notes during the trials.

\subsection{Procedure}

Participants were run individually. At the start of each trial, the participant was given a phone and instructions on how to complete the activity. The instructions were read from a script by one of the experimenters. Fourteen of the 28 participants were given training. Training included describing to the participant how the technology worked. The participant was introduced to the phone and told how the tags were to be read, including details of where the readers were in the phones, as well as optimum distances and angles for tag reading. The experimenter demonstrated how to read a tag using the reader on the phone. The trained participants were also given practice runs with a practice poster.

The 14 untrained participants were told only that the reader was on the back of the phone (i.e. the camera or the built-in NFC reader). They were instructed to read the target tag as quickly as possible and move on to the next tag when instructed by a message on the phone's display.

Each participant started at poster 5 and obtained the number of the next poster by scanning the appropriate tag. For example, reading the target tag on poster 5 displayed the message "Go to poster 2". When the target tag was successfully read, the participant read aloud the number of the poster they were to go to next and moved on. The target tags instructed each participant to read the tags in the order $5>2>11>7$ $>4>12>10>6>9>1>3>8$. The target tag on poster 8 instructed the participant 
that she was finished. An error was recorded whenever the participant successfully read a tag that was not the current target tag. In this case, the participant continued attempting to read the target tag until it was successfully read.

After a participant had traversed all 12 posters, she completed a questionnaire. Training in the other tagging technology was then given (dependent on the trained/untrained condition) and the trial was repeated with the other tagging technology. The order in which the tagging technologies were used was counter-balanced. After each participant completed her trials with both tagging technologies, she was given a third questionnaire to compare her experiences of the two technologies.

\subsection{Experimental results}

The dependent variable was the cumulative time taken from the first tag being read on poster 5 to the target tag on the final poster being read. Figure 5 shows the total times (in seconds) for NFC and 2D barcode use by the untrained participants.

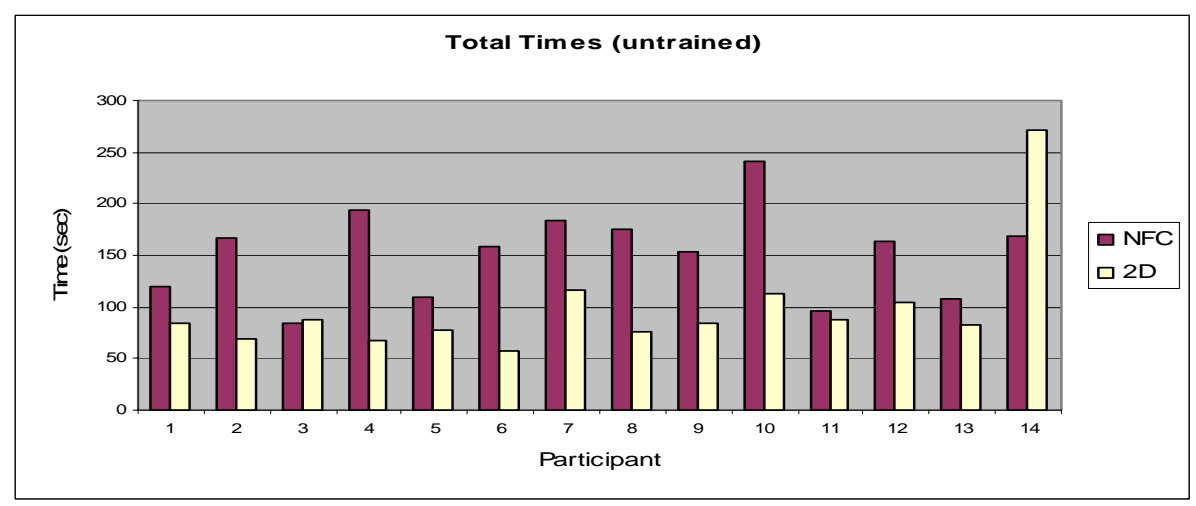

Fig. 5. Times (in seconds) for untrained participants.

Figure 6 shows the total times (in seconds) for NFC and 2D barcode use by the trained participants.

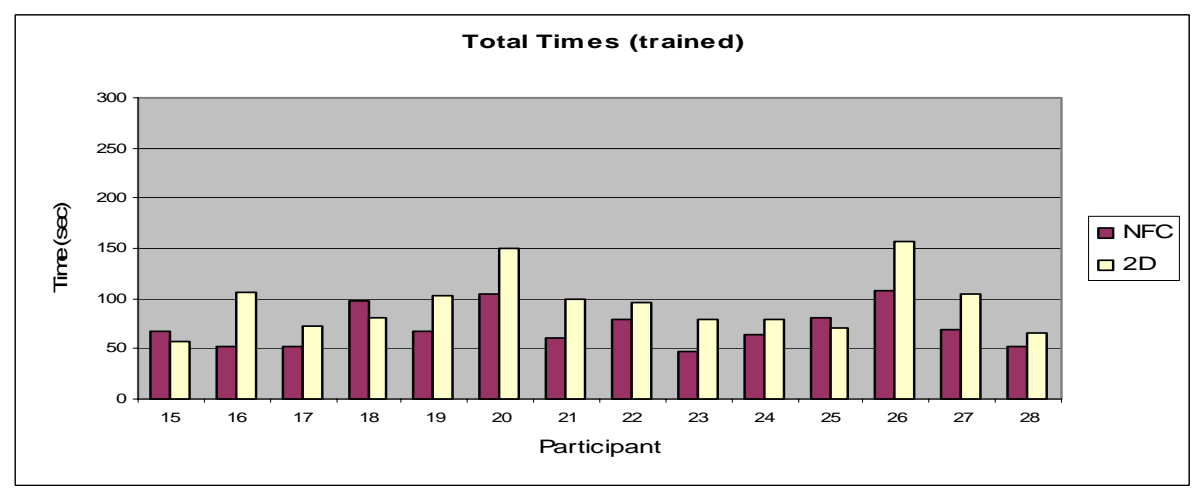

Fig. 6. Times (in seconds) for trained participants. 
Table 3 shows the mean (and SD) time to read all 12 tags using each technology, with and without training.

Table 3. Mean (and SD) time to read all 12 tags for both the 2D barcodes and NFC tags, with and without training.

\begin{tabular}{lll}
\hline & NFC & 2D barcodes \\
\hline Untrained & 151.50 & 98.43 \\
& $(43.33)$ & $(52.42)$ \\
Trained & 71.71 & 94.57 \\
& $(20.01)$ & $(29.18)$ \\
\hline
\end{tabular}

A mixed 2-way ANOVA for the data in Table 3, with tagging technology and training as the independent variables, found a significant effect $\left(\mathrm{F}_{3,24}=16.67, \mathrm{p}=\right.$ 0.000154). In order to determine the direction of the differences, post hoc Tukey tests were performed.

A Tukey test for the main effect of tagging technology found a significant difference between NFC and 2D barcodes for untrained users $(\mathrm{T}=5.18, \mathrm{Q}=4.7, \mathrm{~T}>\mathrm{Q}, \mathrm{P}$ $<0.01$ ), supporting hypothesis 1 , while there was no significant difference between NFC and 2D barcodes for trained users $(\mathrm{T}=2.23, \mathrm{Q}=3.79, \mathrm{~T}<\mathrm{Q}, \mathrm{p}>0.05)$.

When using NFC, there was a significant difference between the training and no training conditions $(\mathrm{T}=7.79, \mathrm{Q}=4.7, \mathrm{~T}>\mathrm{Q}, \mathrm{p}<0.01)$, supporting hypothesis 2 , while there was no significant difference between the training and no training conditions when using 2D barcodes, $(\mathrm{T}=0.38, \mathrm{Q}=3.79, \mathrm{~T}<\mathrm{Q}, \mathrm{p}>0.05)$.

So, both experimental hypotheses were supported. Without training, using the NFC tags took significantly longer than using the 2D barcodes (H1). With training, users' performance with the NFC tags significantly improved (H2), to the point at which there was no difference between using 2D barcodes and NFC. Training had no effect on users' performance with 2D barcodes.

The observations of the trials, the video recordings and the post-trial questionnaires provided supporting qualitative data. On a Likert scale (1: very difficult, 5: very easy), the untrained participants found it easier to use 2D barcodes than NFC. This finding was consistent across the immediate post-trial questionnaires (NFC: 3.8, 2D barcode: 4.6 ) and the third questionnaire (NFC: 3, 2D barcode: 4.1 ). When asked which phone they would pick if they had to repeat the task, almost all the untrained participants stated that they would prefer to use the 2D barcode technology. Not knowing which part of the phone to touch on the tag was the main frustration reported with the NFC phone.

In contrast, the trained participants found it easier to complete the task using NFC rather than the $2 \mathrm{D}$ barcodes. Again, this was consistent across the immediate posttrial questionnaires (NFC: 4.1, 2D barcode: 3.9) and the third questionnaire (NFC: 4.1, 2D barcode: 3.9). When asked which phone they would pick if they had to repeat the task, almost all the trained participants stated that they would prefer to use the NFC technology. The main complaint about the 2D barcode phone was the re- 
quirement to align the phone with the 2D barcode, in contrast to just touching the tag with the NFC phone.

When asked in the third questionnaire "Which technology did you find easier to use?”, only 3 of the 14 untrained participants said they found NFC easier, while 11 of the 14 trained participants said they found NFC easier.

Video analysis showed that while many untrained users struggled to use the NFC phone, many developed more or less successful techniques in their attempts to get the device to work. Some users made quick stabbing motions at the tag, rather than holding the phone over the tag until it was read. Such interactions meant that many attempts were needed to read the tag and this contributed to increasing the overall time taken. Some participants carefully held the phone horizontally, apparently believing that this facilitated tag reading.

One participant found it extremely difficult to read any 2D barcodes on the bottom row of posters. The video record suggested that his problems were due to not holding the phone at an appropriate angle to compensate for aiming the phone's camera downwards (thereby moving it away from parallel to the plane of the tag).

Posters 3, 7 and 11 caused particular problems for the untrained participants using NFC, with between 20 and 30 errors (i.e. reading non-target tags) for each of these posters. The arrangement of tags on these posters was that shown in Figure 5 (b). This arrangement led to participants repeatedly reading the wrong tag. Training reduced the errors, contributing in turn to significantly reduced task completion times.

\section{Conclusions and future directions}

Our field trial and our experimental pilot study suggested that users were unsure of the location of the NFC reader in the mobile phone. In the field trial and the experimental evaluation, users developed their own techniques for successful tag reading, although these often involved manipulating or holding the phone in ways that were not actually necessary. Our experimental evaluation tested the hypothesis that an initial training period would overcome users' lack of familiarity with the NFC technology, leading to better performance. This was found to be the case. Initial training had no effect on users' performance with the $2 \mathrm{D}$ barcodes. It seems likely that this is partly due to users' previous familiarity with cameraphones, and partly due to the obvious location of the camera on the phone and the visual feedback on the display of the camera image. These factors are likely to have contributed to users' performance being at a level that was unaffected by the brief training offered. It is likely that longer term familiarity with NFC would produce much the same effect for that technology.

Participants in the field trial reported feeling awkward or embarrassed reaching out to use the tags in a public place. Similarly, in a user study of service discovery and interaction using mobile phones and RFID tags, [9] found that users were reluctant to use this from of interaction outside their own homes "because they were embarrassed about touching RFID tags in public places. For example, they felt that touching might seem too eye-catching on the street or at a shopping mall. So, although touching is itself a natural action, it isn't necessarily considered a socially acceptable way 
to use new technology” [p. 45]. However, even during the limited course of our field trial, participants - and their colleagues - became more accustomed to tag-reading in public. Not so long ago, voice calling and texting in many public places would have seemed unusual but they have rapidly become appropriated. There is still a frisson of the weird about seeing a user of a discreet hands-free headset wander along, talking animatedly apparently to thin air, but that too is rapidly becoming a commonplace. It seems unlikely that it will take long for public tag reading to be similarly appropriated.

[9] also found that users were unwilling to read tags in public places "because they considered the security risk too high” [p. 45]. Again, this was reflected in our participants' concerns. Mobile phone theft accounts for about half of all street crime in the UK (http://www.met.police.uk/mobilephone/) but there is evidence that high traffic places are often safer than low traffic places [3], suggesting that the actual threat may be perceived differently from the real threat. Reading a tag may be slightly more risky than calling or texting in a public place only in so far as one is stationary while reading the tag. Along with the issue of long interaction times blocking others' access both to the place and to the tag, this would suggest designing to minimize interaction time with the tag, retaining most of the interaction on the phone via a wireless connection to services initially identified by the tag reading everihthe concern of some users about unintentional interactions with NFC tags suggests that users should have more control of the NFC functionality so that, at least, they can turn it on and off when desired. The issues of impersonating others and rewriting tags may suggest a requirement for lockable or "read-only" NFC tags. However, disabling write access to a tag would disable some of the intrinsic advantages of NFC. 2D barcodes, at least in their printed form, will require a different approach to security since they may be overlaid or replaced quite easily.

In proposing access control as an application of NFC, our participants voiced no concerns about its potential role in monitoring their movements. This is in contrast to the commonly reported fears of privacy being eroded by tagging systems [2]. Whether or not a tag based, or indeed swipe card based, access control system logs events is a design decision. Such decisions and users' acceptance of such monitoring will depend at least in part on how much they value the services offered.

Our participants' most commonly suggested application of exchanging contacts data by touching 2 phones together is the functionality that raises NFC beyond a simple tagging technology. In [5] we report an application that does this, comparing the contrasting influences on the user experience of using NFC and Bluetooth. The changing costs of tags and barcode production, the production of phones with appropriate readers and, ultimately, the development of useful, usable applications and services will influence the trajectory of tagging technologies, but it seems likely to be upward. 
Acknowledgements. We thank Nokia, and Jason Cooper, Nilusha Piyasena, Trevor Richards, Mickael Vaillant, Craig Webb and Jan Kietzmann.

\section{References}

1. Garfinkel, S.L., Juels, A. and Pappu, R. (2005) RFID privacy: an overview of problems and proposed solutions. IEEE Security \& Privacy 3 (3), 34-43.

2. Günther, O. and Spiekermann, S. (2005) RFID and the perception of control: the consumer's view. Commun. ACM 48 (9), 73-76.

3. Hillier, B. and Shu, S. (2000) Crime and urban layout: the need for evidence. In: Ballityne, S., Pease, K. and McLaren, V., Secure foundations: key issues in crime prevention, crime reduction and community safety, London, IPPR.

4. Kindberg, T. and O’Hara, K. (2006) "You are here" - experiences with located systems. HP Labs Technical Report HPL-2006-120. http://www.hpl.hp.com/techreports/2006/HPL2006-120.pdf.

5. Kostakos, V., O’Neill, E. and Shahi, A. (2006) Building common ground for face to face interactions by sharing mobile device context. LOCA 2006, LNCS 3987, 222-238.

6. Ohkubo, M., Suzuki, K., and Kinoshita, S. (2005) RFID privacy issues and technical challenges. Commun. ACM 48 (9), 66-71.

7. Patel, S.N., Kientz, J.A., Hayes, G.R., Bhat, S. and Abowd, G.D. (2006) Farther than you may think: an empirical investigation of the proximity of users to their mobile phones. Ubicomp 2006, Springer LNCS 4206, 123-140.

8. Pering, T., Ballagas, R., and Want, R. (2005) Spontaneous marriages of mobile devices and interactive spaces. Commun. ACM 48 (9), 53-59.

9. Riekki, J., Salminen, T. and Alakarppa, I. (2006) Requesting pervasive services by touching RFID tags. IEEE Pervasive Computing, 5 (1), 40-46.

10. Rukzio, E., Leichtenstern, K., Callaghan, V., Holleis, P., Schmidt, A. and Chin, J. (2006) An experimental comparison of physical mobile interaction techniques: touching, pointing and scanning. Ubicomp 2006, Springer LNCS 4206, 87-104.

11. Scott, D., Sharp, R., Madhavapeddy, A., and Upton, E. (2005) . Using visual tags to bypass Bluetooth device discovery. SIGMOBILE Mob. Comput. Commun. Rev. 9, 1 (Jan. 2005), 41-53.

12. Toye, E., Sharp, R., Madhavapeddy, A. and Scott, D. (2005) Using smart phones to access site-specific services. IEEE Pervasive Computing 4 (2), 60-66.

13. Want, R. (2006) An introduction to RFID technology. IEEE Pervasive Computing JanMar 2006, 25-33. 